\title{
Incarcerated diaphragmatic hernia and cardiac tamponade
}

\author{
Kevin J. McKeown, MD • William Sischek, MD
}

Received: 14 June 2010/ Accepted: 16 November 2010/Published online: 4 December 2010

(c) Canadian Anesthesiologists' Society 2010

\section{To the Editor,}

We present an unusual cause of tamponade-like presentation in a patient undergoing anesthesia. We briefly discuss the related pathophysiology and the need for vigilance in maintaining a broad differential diagnosis for critical events occurring in the operating room (OR). The patient gave written consent for publication of this report.

A 76-yr-old male presented to hospital with sudden acute onset of abdominal pain and nausea. He was hemodynamically stable in the emergency department where blood tests revealed an elevated lactate level (3.4 $\left.\mathrm{mmoL} \cdot \mathrm{L}^{-1}\right)$. Computed tomography of the patient's abdomen showed a left diaphragmatic hernia defect and small bowel herniation. He was treated with antibiotics and fluid resuscitation by the surgical team while a plan for surgery was being formulated with the concern for potential bowel ischemia. The patient's condition acutely deteriorated on the surgical floor. He developed increasing oxygen requirements and became hypotensive. Repeat investigations showed a serum lactate of $10.5 \mathrm{mmoL} \cdot \mathrm{L}^{-1}$ with a blood $\mathrm{pH}$ of 7.19. The patient was transferred to the OR for emergency exploratory laparotomy and possible left thoracotomy for reduction of a suspected incarcerated paraesophageal hernia with bowel ischemia.

On initial examination outside the OR, the patient was in obvious respiratory distress on $15 \mathrm{~L}$ oxygen with a nonrebreathing mask, and there were visible signs of air hunger with oxygen saturations in the 80 's. He was immediately transferred into the OR, and aggressive fluid resuscitation was initiated while preparing to secure the patient's airway emergently. Rapid sequence induction was performed with

K. J. McKeown, MD $(\bowtie) \cdot$ W. Sischek, MD

University of Western Ontario, London, ON, Canada

e-mail: kevin.mckeown@utoronto.ca propofol $30 \mathrm{mg} i v$, succinylcholine $120 \mathrm{mg} i v$, and tracheal intubation.

The patient remained hypotensive with a systolic blood pressure of approximately $80 \mathrm{mmHg}$ and a heart rate of 120 beats $\mathrm{min}^{-1}$ despite fluid resuscitation. The initial central venous pressure (CVP) was $20 \mathrm{mmHg}$, and the peak airway pressures reached $40 \mathrm{~cm} \mathrm{H}_{2} \mathrm{O}$. An epinephrine infusion was initiated at a rate of $0.1 \mu \mathrm{g} \cdot \mathrm{kg}^{-1} \cdot \mathrm{min}^{-1}$.

As surgery progressed and proceeded from a laparotomy to a thoracotomy, there was an audible decompression of the herniated bowel from the chest with an immediate resolution of the high airway pressures, tachycardia, and elevated CVP. The patient's blood pressure and heart rate normalized, his urine output improved, and he was able to be weaned from the epinephrine infusion. The patient tolerated the remainder of the procedure well, and he was transferred to the intensive care unit. Subsequent review of his immediate preoperative chest $x$-ray showed left chest opacification and bowel herniation with right mediastinal shift (Fig. 1).

The pathophysiology of this case can best be described as the patient having an incarcerated ischemic bowel in the chest producing cardiac compression and tamponade-like physiology. Reduction of the herniated bowel resulted in the immediate resolution of the patient's hemodynamic instability. The patient's postoperative chest $x$-ray showed resolution of the mediastinal shift (Fig. 2).

Cardiac tamponade is a life-threatening slow or rapid compression of the heart due to the pericardial accumulation of fluid, pus, blood, clots, or gas resulting from effusion, trauma, or rupture of the heart. ${ }^{1}$ In this case, we believe that the incarcerated bowel became ischemic and edematous, causing extrinsic compression and displacement of the heart and resulting in cardiogenic shock. The $x$-ray of the patient at original presentation to the hospital 


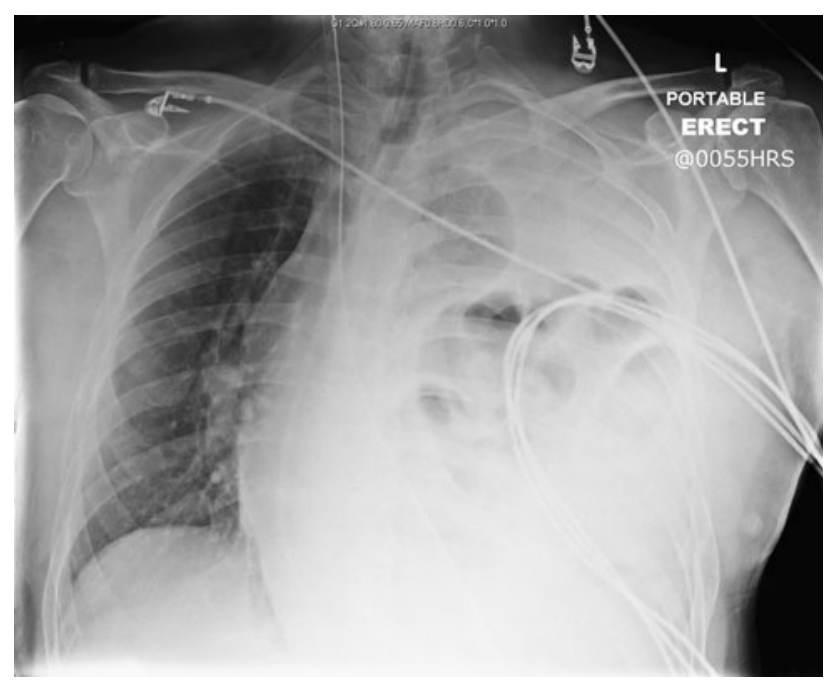

Fig. 1 Portable upright chest $x$-ray of the patient after acute decompensation on the ward. The $x$-ray shows significant rightward mediastinal shift from an incarcerated bowel

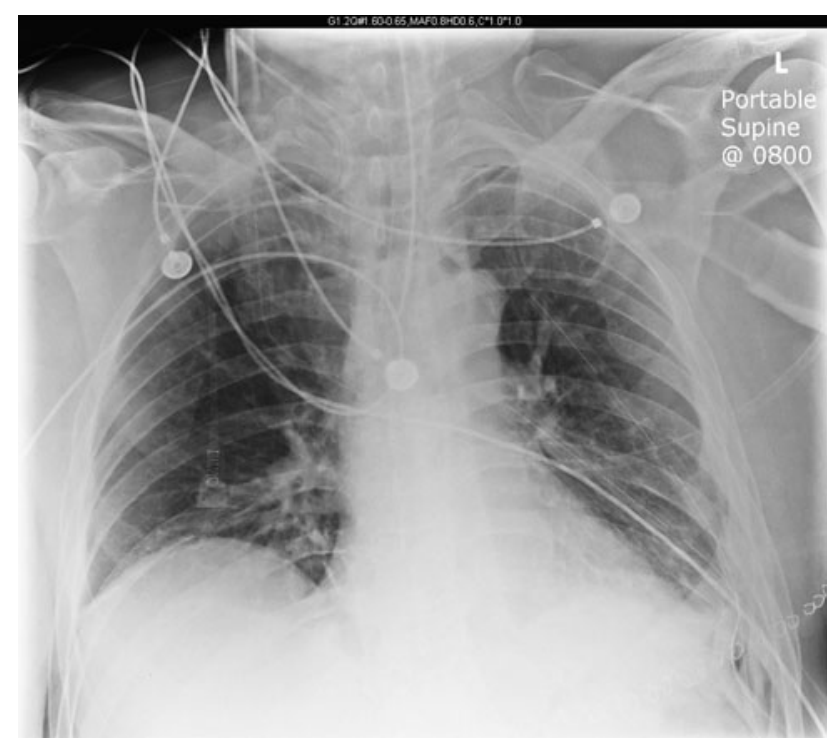

Fig. 2 Portable upright chest $x$-ray of the patient after surgical decompression of the incarcerated bowel. The $x$-ray shows resolution of the mediastinal shift

when he was hemodynamically stable did not show evidence of mediastinal shift.

Tamponade is usually diagnosed both clinically and by echocardiographic imaging, and it is characterized by dyspnea, tachycardia, pulsus paradoxus, elevated jugular venous pressure, and hypotension. ${ }^{1,2}$ It has been stated that dyspnea at rest that progresses to air hunger at rest is a key feature of tamponade. ${ }^{1}$ In fact, our patient had every clinical feature of tamponade. Unfortunately, we were exposed to the patient late in the progression of his disease, and we were unable to check for pulsus paradoxus. Based on the patient's history given to us by the surgical team, we had no information that would make us suspicious of a tamponade-like physiology for the diagnosis of his shock. No echocardiography was performed, which may have shown right atrial or ventricular chamber collapse characteristic of tamponade. $^{3}$

Cardiac compression leading to tamponade-like physiology is reported in the literature. A recently published case involved a patient who developed cardiogenic shock after cardiac surgery. The patient returned to the OR, but no pericardial collections were found. Eventually, it was discovered that the patient had a hiatus hernia and that his stomach had become distended in the thorax causing compression of the heart. ${ }^{4}$ Another recent case highlights the importance of extrinsic compression of the heart causing tamponade-like physiology. ${ }^{5}$ It was found that the tamponade-like physiology was caused by a large leftsided pleural effusion, and the ensuing drainage of the effusion resulted in the immediate resolution of symptoms. These cases highlight the importance of suspecting tamponade-like physiology even when there does not appear to be the classic etiology involving pericardial disease.

Competing interests None declared.

\section{References}

1. Spodick DH. Acute cardiac tamponade. N Engl J Med 2003; 349: 684-90.

2. Roy CL, Minor MA, Brookhart MA, Choudhry NK. Does this patient with a pericardial effusion have cardiac tamponade? JAMA 2007; 297: 1810-8.

3. Settle HP, Adolph RJ, Fowler NO, Engel P, Agruss NS, Levenson NI. Echocardiographic study of cardiac tamponade. Circulation 1977; 56: 951-9.

4. Devbhandari MP, Khan MA, Hooper TL. Cardiac compression following cardiac surgery due to unrecognised hiatus hernia. Eur J Cardiothorac Surg 2007; 32: 813-5.

5. Kopterides $P$, Lignos $M$, Papanikolaou $S$, et al. Pleural effusion causing cardiac tamponade: report of two cases and review of the literature. Heart Lung 2006; 35: 66-7. 